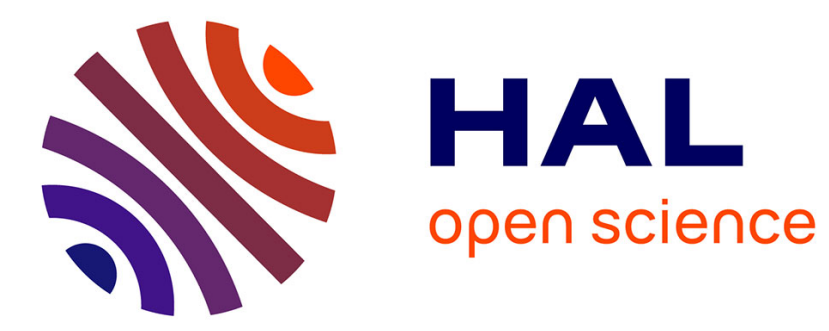

\title{
Dynamic Lifting by Whole Body Motion of Humanoid Robots
}

Hitoshi Arisumi, Sylvain Miossec, Jean-Rémy Chardonnet, Kazuhito Yokoi

\section{To cite this version:}

Hitoshi Arisumi, Sylvain Miossec, Jean-Rémy Chardonnet, Kazuhito Yokoi. Dynamic Lifting by Whole Body Motion of Humanoid Robots. IROS'08: International Conference on Intelligent Robots and Systems, Sep 2008, Nice, France. pp.668-675. lirmm-00536305

\section{HAL Id: lirmm-00536305 https://hal-lirmm.ccsd.cnrs.fr/lirmm-00536305}

Submitted on 15 Nov 2010

HAL is a multi-disciplinary open access archive for the deposit and dissemination of scientific research documents, whether they are published or not. The documents may come from teaching and research institutions in France or abroad, or from public or private research centers.
L'archive ouverte pluridisciplinaire HAL, est destinée au dépôt et à la diffusion de documents scientifiques de niveau recherche, publiés ou non, émanant des établissements d'enseignement et de recherche français ou étrangers, des laboratoires publics ou privés. 


\title{
Dynamic Lifting by Whole Body Motion of Humanoid Robots
}

\author{
Hitoshi Arisumi, Sylvain Miossec, Jean-Rémy Chardonnet, and Kazuhito Yokoi, Member, IEEE
}

\begin{abstract}
A motion control method of lifting a heavy object up to a higher position with humanoid robots is developed. The key issue of lifting motion is how to reduce the load on humanoid arms in which low-power actuators are implemented. The use of singular postures of arms is well-known to avoid actuator saturation of the arms. By combining two different kinds of humanoid motions such as accelerating an object upward and sliding the body into under the object, we propose a method that enables to transit one singular posture of arms to another while lifting the object. Simulation results show the effectiveness of the proposed method for reducing the load on the arms. We realize a motion of lifting a heavy object dynamically with the humanoid robot HRP-2 through experiment.

Index Terms - Humanoid robot, Dynamic whole body manipulation, ZMP, Lifting
\end{abstract}

\section{INTRODUCTION}

$\mathrm{M}$ ANIPULATION of objects by fixed-base robots is a research field that is widely explored. For instance, high speed manipulation by industrial robots, parallel manipulators with high power output actuators used to move heavy objects are some examples of technologies that have been developed. There are other works dealing with object manipulation such as control of a rolling object placed on arms [1] or a rolling ball placed on butterfly shaped arms [2]. As these robots have a fixed base, it is therefore very simple to compare the motions with the reality.

On the other hand, there are some works on manipulation by non-fixed base robots such as humanoid robots, for instance, collaborative manipulation with humans [3] or pushing manipulation [4]. In these works, light objects or objects for which weight is not needed to support are considered. In object manipulation by a humanoid robot, several methods have been proposed to control the posture of the robot so that it can lift an object statically by keeping the GCoM (grand projection of the center of gravity) of the whole system (robot+object) inside the support polygon [5], [6]. However, these methods do not consider a dynamic lifting of an object grasped by hands to a high position. The RI-MAN robot [7] designed for nursery is able to hold a dummy doll. Built on wheels to ensure a better stability, it hardly however lifts objects using its whole body dynamics.

Manuscript received February 22, 2008. This work was supported in part by the Japan Society for the Promotion of Science under Grants-in-Aid for Scientific Research (B) 17360120.

H. Arisumi (Corresponding Author), S. Miossec, J-R Chardonnet, and K. Yokoi are with the Intelligent Systems Research Institute of the National Institute of Advanced Industrial Science and Technology (AIST), Tsukuba Central 2, 1-1-1 Umezono, Tsukuba, Ibaraki 305-8568, Japan. Contact TEL: +81-29-861-7282 FAX: +81-29-861-3443 e-mail: h-arisumi@aist.go.jp
In some other works dealing with lifting manipulation with human beings, rational torque patterns of a skilled weightlifter [8] and the relationship between speed and lifting postures [9] are analyzed. However, these data are not still applied to the lifting manipulation with a robot maybe because its structure such as mechanism or output performance is different from human's one. Another work considers the weightlifting of the Puma robot [10] taking into account the dynamic model. The weightlifting motions are obtained through an optimization process considering B-splines parameterized motions. Joint position, velocity and torque limits are considered. However, they did not consider a good enough actuator model and a joint dry friction. Additionally, they had no issue of stability for their fixed-based robot.

In our earlier works, we focused on a preliminary motion of a robot before manipulation, and proposed to use it for dynamic lifting by impulsive forces [11]. We performed experiments on a real humanoid robot, and we confirmed the feasibility of lifting a box placed initially on the floor at the height of the waist of the robot, showing the effectiveness of the method. However, there is a possibility that the actuators get saturated when the robot lifts a heavy object because their characteristics are not considered.

Our goal in this paper is to realize a dynamic motion of lifting an object above the head of the humanoid robot in consideration of materials handling operation of the robot as shown in Fig. 1. We thus address how to move the object from initial sate to final one passing through the unstable area in which the robot cannot hold the object statically due to saturation of the actuators. To solve the problem, we propose a motion planner based on an optimization method with making better use of actuator's characteristics. We confirm the availability of the proposed method through simulations and experiments.

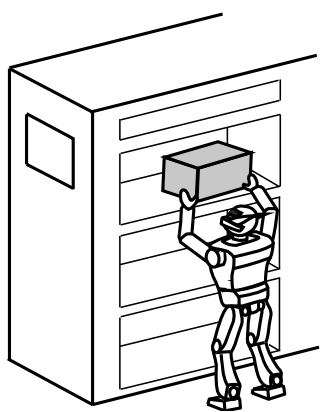

Fig. 1. Materials handling operation.

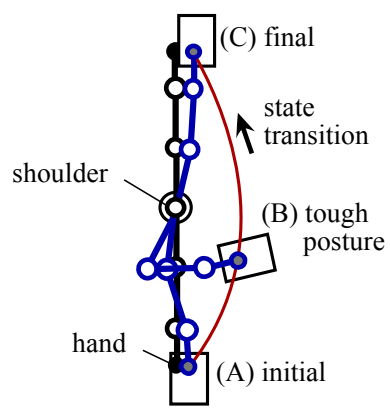

Fig. 2. Singular configuration of arm. 


\section{STRATEGY FOR DYNAMIC LIFTING A HEAVY OBJECT}

\section{A. Configuration of low power output joints}

When the robot carries a heavier object, generally higher joint-torques is needed. Even in the case that an object is light and large, higher joint-torques may be needed because the object is carried far away from the base of the robot in the horizontal direction. Those may cause a saturation of joint-output. Therefore, the static reachable space of robot's hand with an object is restricted by its weight or size. Moreover in the case of unfixed robot, the static reachable space of the hands is restricted by the projection of the center of mass (CoM) of the whole system, robot and object, as well as weight or size of object.

In order to keep away from the torque limits, a singular configuration is effective for low powered parts of the robot to withstand the load of the object. In the case of human beings, for example, we often take a way to receive the load of object mechanically by making the arm closer to a singular configuration instead of applying force to an object actively. A specification of actuator of the robot, HRP-2 robot made by Kawada Industries, is shown in Table I [12]. The output limits of the wrist and the elbow are quite smaller than that of other parts of the robot, which is similar to human beings.

TABLE I

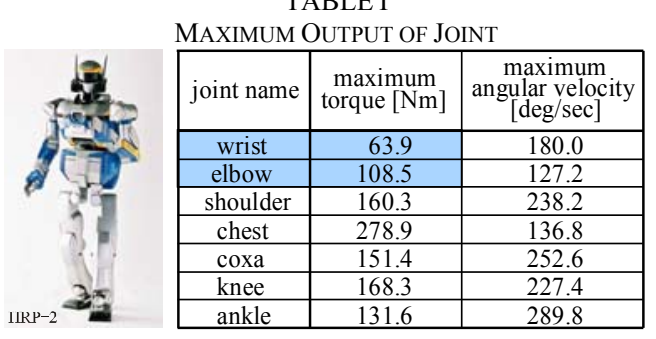

We can consider two singular postures of arm in the vertical plane, namely stretched arm as shown in Fig. 2 (A) and (C). Those postures allow the robot to keep holding an object statically. When the posture is closer to singular, we can expect that the robot can carry a heavy object.

One of the important key issues of this work is to develop the method of transit dynamically from one static stable state to the other passing through static unstable state such as a configuration (B) in Fig. 2.

In this paper, we investigate how to reduce the load on the hands by using a whole body motion of the robot to lift an object from initial configuration (A) to final configuration (C) so that a heavy object can be held at a high position. We challenge the control of lifting a heavier object dynamically at once in the case that it is impossible to do it statically.

\section{B. Method to change the posture of the arm}

To go from singular posture (A) to another singular posture (C), we would like to review two methods. For each method, we point out the different problems.

$<$ Transition I $>$ As shown in Fig. 3a, when a singular configuration is reached, we ask the robot to stand up or tilt backward which is easily done thanks to the high power output in the leg parts and in the waist. By using the inertia of the object which will thus gives an acceleration, we reduce the drive power needed to support the load of the object, as shown in Fig. 3b, we stretch the arm and so we go to the other singular configuration (Fig. 3d).

(Problem) If the mass of the object is big and considering the torque limits of other parts than those of the arm, it can lift the object until the position marked in Fig. 3c, meaning that it cannot stretch the arm anymore, thus it becomes impossible to keep holding the object.

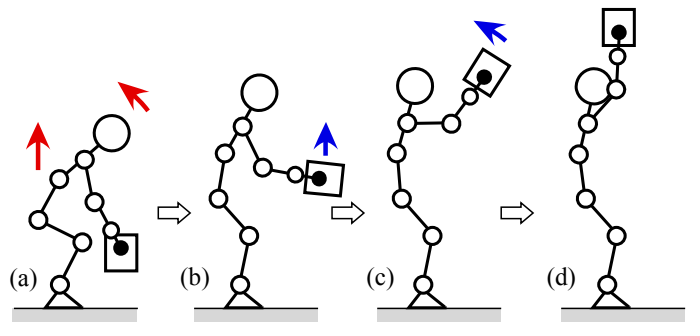

Fig. 3. State transition of arm by using inertia of object.

(Transition II) We ask the robot to go from the statically stable position of Fig. $4 \mathrm{a}$ to the one of Fig. $4 \mathrm{~b}$, which means to crouch quickly under the object and to reach a position where it can keep holding the heavy object (Fig. 4c).

(Problem) As the robot will crouch under the object then stretch its arm, it is necessary to control very quickly the postures of the robot to be faster than the fall of the object that will be caused by this change of posture.

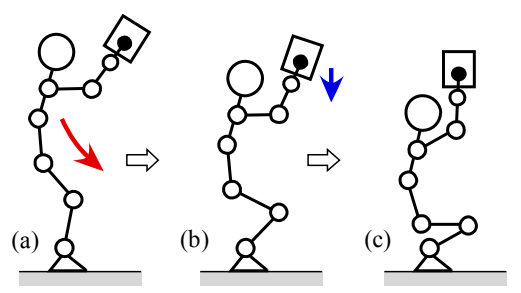

Fig. 4. State transition of arm by changing configuration of whole body.

\section{Method to lift the object}

If we combine the two previously presented approaches Transitions I and II, we think that the problems will compensate each other. In other words, if we design a lifting motion with method I, we give to the robot enough time and space to crouch under the object with method II and so we do not have to define many changes of posture. Moreover, if we allow the robot to reach a stable position for which it can keep holding the object with method II, when it will stand up with method I, it will not need to lift the object to a high position to stretch its arm. As shown in Fig. 5, the combination of methods I and II, which will be named method III, is expected to reduce the load on the arms with the object moving upward and allow the robot to stretch its arms by crouching under the object.

In this paper, we will design a control law that lets the robot to stand up from a position defined in Fig. 5a, then switch 
(Fig. 5c) from lifting (Fig. 5b) to crouching (Fig. 5d) while lifting the object. Finally, it will reach the standing up position in Fig. 3d from the sitting position (Fig. 5e), so that the configuration becomes nearly singular.

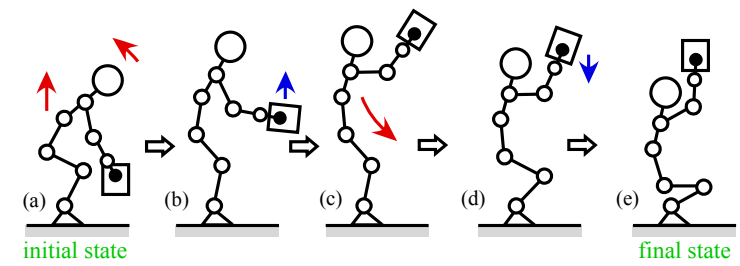

Fig. 5. Target motion.

\section{DESIGN OF TRAJECTORY}

\section{A. Model}

In this paper, we assume that the system is symmetric during manipulation. Hence from a 30-dof humanoid robot model, we simplify it to a 7-dof model with 8 links. As shown in Fig. 6, we set the coordinate system so that $\mathrm{x}$-axis is on the floor and z-axis passes through the ankle joint. $\mathrm{J}_{i}$ represents the joint $i, \varphi_{o b j}$ represents the angle between the horizontal line and the hand, and $F_{h}$ represents the external force at the grasping point. The robot's mass and the object's mass are noted as $m_{r o b}, m_{o b j}$ respectively, the inertia moment around the center of mass of the object is noted $I_{o b j}$ and their values are known. Furthermore, $g$ denotes the gravity acceleration. The center of mass of the object is located at the middle point of the two grasping points.

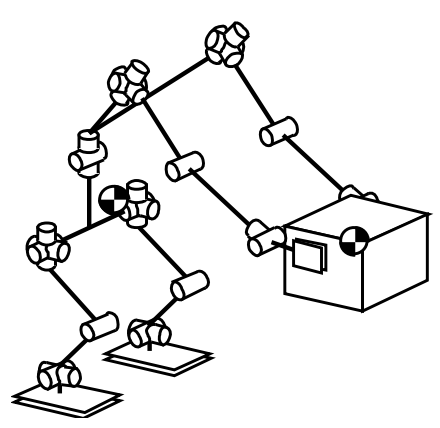

(a) 30-d.o.f. model

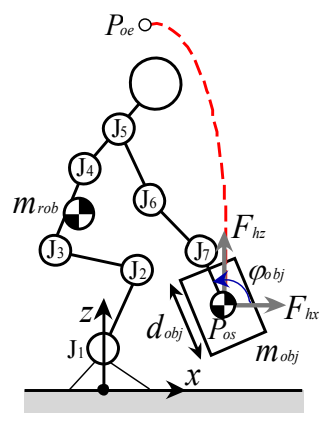

(b) 8 link-model
Fig.6. Humanoid robot model.

\section{B. Characteristics of the joint output}

The actuator's output is generally limited by the torques and the angular velocity. In the case of the DC motor that is widely used for robots, its possible output is derived from the limits of the input voltage and the limits of the electric current of the armature [13]. Therefore, the relationship between the angular velocity $\dot{\theta}_{j}$ and the torque $\tau_{j}$ of the joint $j$ can be written as follows:

$$
\begin{gathered}
\left|\dot{\theta}_{j} / \dot{\theta}_{\text {max }}+\tau_{j} / \tau_{\text {jmax }}\right|<1 \\
\left|\tau_{j}\right|<\tau_{\text {jmax }}
\end{gathered}
$$

where $\dot{\theta}_{\text {max }}$ and $\tau_{\text {jmax }}$ are the maximum angular velocity and the maximum torque of the joint $j$, respectively. Their values are shown in Table I. Characteristics of the DC motor itself are expressed by the inequalities which are given by substituting the following equations into (1) and (2).

$$
\dot{\Theta}=\dot{\Theta}_{M} / n_{g}, \Gamma=n_{g} \Gamma_{M},
$$

where $\dot{\Theta}=\left[\dot{\theta}_{j}, \dot{\theta}_{\text {max }}\right]^{\mathrm{T}}, \dot{\Theta}_{M}=\left[\dot{\theta}_{w j}, \dot{\theta}_{M \text { jimax }}\right]^{\mathrm{T}}, \Gamma=\left[\tau_{j}, \tau_{\text {jmax }}\right]^{\mathrm{T}}, \Gamma_{M}=$ $\left[\tau_{M j ;}, \tau_{\text {Mjimax }}\right]^{\mathrm{T}}, n_{g}$ is the reduction gear ratio, and subscript " $M$ " describes the motor.

It should be noted that the DC motor deteriorates its performance or burns out in the worst case if it keeps running over the rated output and the allowable time. In this paper, $\Delta t_{m o t}$ denotes the allowable time. On the other hand, the motor can keep running for a long time if its output is within the rated output. Moreover, note that the allowable load of the force sensor mounted in the wrist restricts the maximum output of the wrist joint. We will give these values in Section IV.

\section{Reachable space of hands}

In the initial and final posture of the lifting motion, the robot needs to hold the object statically. We here introduce the static stable reachable space, SsRS [11]. It is defined as the set of positions of the robot's hand when the following constraints are all satisfied.

1) Torque of each joint is within the rated torque, and torque of the wrist is within the allowable load of the force sensor.

2) The GCoM of the whole system, the robot and the object, is included in the support polygon.

3) There is no geometrical interference between the robot and the object.

4) Each joint angle is within each joint limit.

As for constraint 1), the following equation is used for calculation of the joint torque vector $\tau$ in case that the robot can statically support the external force $\boldsymbol{F}$.

$$
\boldsymbol{\tau}=\mathrm{G}(\boldsymbol{\theta})-J^{\mathrm{T}} \boldsymbol{F}
$$

where $\boldsymbol{\theta}$ is the vector of all joint angles, $\mathrm{G}(\boldsymbol{\theta})$ is the torque vector of gravity, and $J^{\mathrm{T}} \boldsymbol{F}$ is the torque vector generated by the external force $\boldsymbol{F}$ that applies to the hands. $J^{\mathrm{T}}$ is the transpose of the Jacobian matrix. Notice that the torque vector $\tau$ obtained by (3) needs to satisfy not with (2) but with the rated torque limitation in order to hold the object statically for a long time.

Giving the angle vector $\theta$, we can get the torque vector $\tau$ and the geometrical information of the robot. If all constraints mentioned above are satisfied, the hand's position calculated by $\boldsymbol{\theta}$ is a point of the SsRS. Repeating that procedure, we finally get the set of points of hand, namely the SsRS. We can select the initial/final position of the hand from SsRS. 


\section{Desired trajectory}

The dynamic lifting motion can be obtained by an approximated optimal control method. The optimal control problem is discretized by regarding joint angles trajectories as B-splines and time-discretizing constraints. The resulting problem is solved with the optimization program IPOPT [14], which is an interior point method. We have already shown the availability of the motion planner based on IPOPT by applying it to the kicking motion of the humanoid robot [15]. We here apply it to the dynamic motion with passing through the area where the robot is statically unstable.

We precisely present the robot dynamic model, the motion parameterization, how the lifting motion is defined, the robot constraints, and the criteria for optimization.

$<$ Dynamic model $>$ We consider the whole dynamic model of the HRP-2, with inertial parameters obtained from CAD model. The inverse dynamic model is used to compute the torques needed to realize a parameterized joint angle motion. The joint dry and viscous frictions are considered to compute the torques. They were experimentally identified as shown in Table II. The object lifted is modeled by additional mass and inertia in each hand. In addition to [15], we here consider a model with closed loop (both feet are on the ground for the lifting).

TABLE II

DRY FRICTION AND VISCOUS FRICTION OF JOINTS

\begin{tabular}{|l|c|c|}
\hline & $\begin{array}{c}\text { dry friction } \\
{[\mathrm{Nm}]}\end{array}$ & $\begin{array}{c}\text { viscous } \\
\text { friction [Nms] }\end{array}$ \\
\hline J1: Ankle & 4.89 & 7.32 \\
\hline J2: Knee & 12.3 & 19.21 \\
\hline J3: Hip & 9.92 & 16.93 \\
\hline J4: Chest & 16.0 & 21.35 \\
\hline J5: Shoulder & 5.32 & 7.22 \\
\hline J6: Elbow & 9.43 & 25.22 \\
\hline J7: Wrist & 5.54 & 10.3 \\
\hline
\end{tabular}

$<$ Motion parameterization $>$ The infinite number of possible lifting motions are reduced to B-splines in the joint space. Therefore, joint angles $\theta_{j}$ are defined by:

$$
\theta_{j}(p, t)=\sum_{i=0}^{n_{b}} p_{i j} B_{i}(t)
$$

where $\mathrm{p}$ are the $\mathrm{B}$-splines parameters, $B_{i}(t)$ the $\mathrm{B}$-splines basis functions and $n_{b}$ the number of $\mathrm{B}$-splines basis functions per joint. For the lifting motion, we used $n_{b}=15$. For the case of closed loop with both feet on the ground, the lost of 6 degrees-of-freedom for one leg is compensated for the possibility to choose the 6 components of force on one foot. Therefore the 6 forces components of the right foot are also specified as B-splines.

$<$ Motion definition $>$ By placing the soles backward and forward, the support polygon gets wider in the $\mathrm{x}$-direction. Indeed the stability in the $\mathrm{x}$-direction may get better in this case, but the robot cannot grasp an object at the low position close to its own legs because of the geometric interference. Therefore, we assume that the robot lifts an object with placing its soles neatly side by side as shown in Fig. 6 that is the same as one lifting style of the weightlifting sport, "the snatch". We take a severer condition of stability to address the challenging issue.

The software presented in [15] for the optimization of motions for HRP-2 has been developed for full-body motions. Here the motion is restricted to the sagittal plane. Joints not participating in the lifting motion are constrained to be zero.

$<$ Constraints $>$ The constraints implemented in the motion optimization must be satisfied with the constraints 3) and 4) described in Section III-C and the following:

1) The constraints of joint output defined by (1) and (2) must be satisfied, and output of the wrist is within the allowable load of the force sensor.

2) The ZMP of the whole system should be inside the support polygon.

3) The time from the initial state to the final state should be less than the time $\Delta t_{m o t}$.

4) Reaction force on each foot is positive. Each foot does not slide.

$<$ Optimization of the motion $>$ Generally, the output limitation of the DC motors mounted in the robot is reduced by the Joule power loss [16]. In order to make more effective use of the output limitation of the motor, the Joule power loss is needed to be minimized. Indeed we can chose criteria such as the operation time or the angular momentum for optimization freely, but we here focus on the electrical energy consumption $E_{r o b}$ for deriving a higher performance from the humanoid robot. The criteria are given by the following equation:

$$
\mathrm{E}_{\mathrm{rob}}=\int_{0}^{t_{f}} \sum c_{j} \tau_{j}^{2}+\dot{\theta}_{j} \tau_{j} d t
$$

where $c_{j}$ is a coefficient depending on the characteristics of actuator and $\tau_{j}$ is the joint torque. The optimal problem constituted of previously defined criteria and constraints with the proposed B-spline parameterization can be put in the standard form:

$$
\begin{array}{cc}
\min _{p, t_{f}} E_{\text {rob }}\left(p, t_{f}\right) \\
\text { subject to } \begin{array}{l}
g_{\text {eq }}\left(p, t_{f}\right)=0 \\
g_{\text {ineq }}\left(p, t_{f}\right) \leq 0
\end{array}
\end{array}
$$

We solve it with the optimization program IPOPT which iteratively decreases the criteria and improves the satisfaction of the constraints. To improve speed and convergence, we additionally computed the gradients of criteria and constraints with respect to $p$ and $t_{f}$.

We do not discuss how the output limitation of the motor varies with the minimization of the Joule power loss through the optimization in this paper. The detailed analysis of that matter is future works. 


\section{Simulation}

\section{A. Reachable area of the hands}

When lifting an object from bottom to top, its posture changes upside down as shown in Fig. 2. First we consider the two postures of the object, $\varphi_{o b j}=90 \mathrm{deg}$ and $\varphi_{o b j}=-90 \mathrm{deg}$ which are corresponding to initial posture and final posture for lifting. These postures can avoid saturation of the torque limit of wrist which is the lowest because of the force sensor. The allowable torque of the force sensor is $5 \mathrm{Nm}$ around $\mathrm{y}$-axis. The allowable force in the direction of the hand is $200 \mathrm{~N}$ and the allowable force in the direction perpendicular to the hand is $100 \mathrm{~N}$. Considering geometrical constraints and output constraints described in III-C, we calculate the SsRS.

Fig. 7 shows the SsRS in the case that $\left(m_{o b j}, d_{o b j}\right)=(8.4 \mathrm{~kg}$, $0.3 \mathrm{~m})$, and $(33.4 \mathrm{~kg}, 0.1 \mathrm{~m})$. The posture of the object in the upper two areas is $-90 \mathrm{deg}$, and that in the lower two areas is 90deg. The light blue are and the green area are the SsRS when $m_{o b j}=8.4 \mathrm{~kg}$. The other areas are the SsRS when $m_{o b j}=$ $33.4 \mathrm{~kg}$. The rated torque of joints is less than $20 \%$ of maximum one described in TABLE I. The computation time to get the area is less than 11 minutes on a PC Intel Core2 Duo $1.88 \mathrm{GHz}$. Checking the singularity of the end parts of the arm when the hand position is included in the blue are and the light blue area, it is found that a heavier object requires a higher singularity. Hence a singular posture is more necessary to hold a heavier object.

As shown in Fig. 7, the upper area and the lower one are separated due to the limitation of joint angles and torques. When the object's posture $\varphi_{o b j}$ is $180 \mathrm{deg}$, it is found that the SsRS does not exist. Therefore the robot cannot hold the object with keeping its posture horizontally. Since the object's posture cannot avoid being 180deg from bottom to top, the object cannot reach the top by static lifting motion. Consequently the dynamic motion with rotating the object is needed to transit between the two SsRSs.

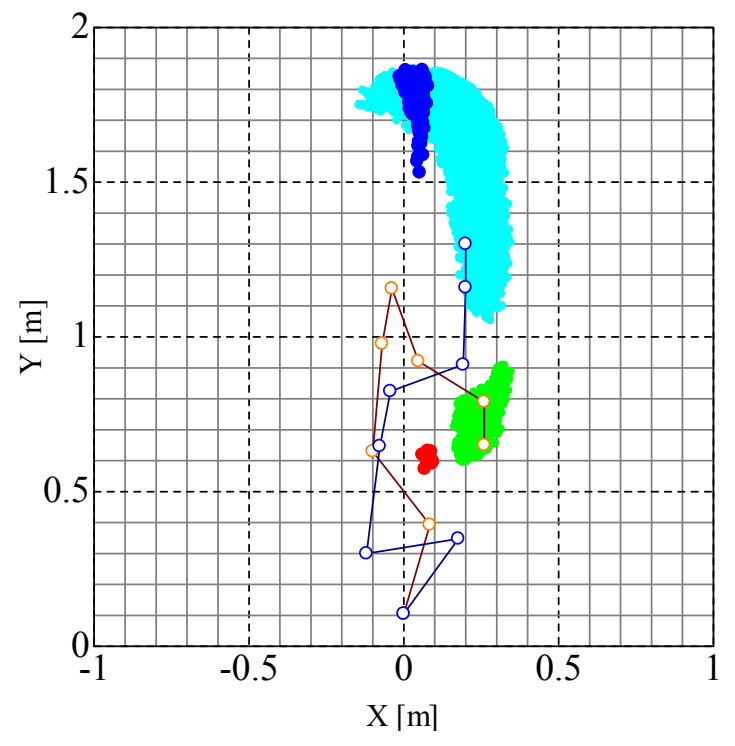

Fig.7. Reachable area of the robot.

\section{B. Dynamic lifting motion}

To prove the validity of our proposal, we make simulation tests on the dynamic simulator that has been especially designed for the humanoid robot HRP-2 [17].

First, we select the initial state and the final state of the robot by using the SsRS in Fig. 7. As described in Section III-C, all joint angles are given and all joint torques are calculated in computing the SsRS. In other words, the posture of the robot corresponding to the hand configuration is given. Then, evaluating the singularity of the arm and the uniform torque distribution in each posture of the robot, we can select the initial and final posture as follows:

\section{$<$ Initial $>$}

Configuration of the object: $(0.26 \mathrm{~m}, 0.65 \mathrm{~m}, 90 \mathrm{deg})$

Positions of joint 3 and 5: $(-0.100 \mathrm{~m}, 0.630 \mathrm{~m})$ and $(-0.039 \mathrm{~m}, 1.156 \mathrm{~m})$

$<$ Final $>$

Configuration of the object: $(0.20 \mathrm{~m}, 1.30 \mathrm{~m},-90 \mathrm{deg})$

Positions of joint 3 and 5: $(-0.120 \mathrm{~m}, 0.300 \mathrm{~m})$ and $(-0.043 \mathrm{~m}, 0.824 \mathrm{~m})$.

These values provide the initial and final postures of the robot by using inverse kinematics. The mass of the object $m_{o b j}$ is $8.36 \mathrm{~kg}$. The object length in the $\mathrm{xz}$-axis direction is $0.15 \mathrm{~m} \times 0.30 \mathrm{~m}$ and has two handles so that the robot can grasp it easily.

Through the optimizing method presented in the previous section, we obtain the trajectory of all joints. In order to improve the convergence of the solution sufficiently, we set the intermediate point which is corresponding to Fig. 5c. The computation time to get the optimal solution is less than 8 minutes on the PC. The duration of the lifting motion is $1.68 \mathrm{~s}$ while the allowable time $\Delta t_{\text {mot }}$ of the DC motors mounted in the humanoid robot is $3 \mathrm{~s}$. The constraint 3 ) described in Section III-D is satisfied.

The blue curves in Fig. 8 show the output of all joints during the whole lifting motion. However, as we supposed our model was symmetric, we show here the output of the one side of leg and arm. Each curve represents the relationship between torques and angular velocities, and the numbers in brackets are the number of the joint. The black polygons are the maximum output limits. These boundary lines are given by Table I, (1) and (2). We can see on this Fig. 8 that the outputs of all joints are within the limits, meaning that it never reaches saturation when lifting the object. We can also remark that the output of the low-powered joints, especially the wrist and the elbow, are controlled to be smaller than that of the other joints.

Next we show in Fig. 9 the force and the moment that appear at the hand. The red curves represent the force in the direction of $\mathrm{x}$-axis and $\mathrm{z}$-axis, and the moment, respectively. The first vertical broken line on the left of the figure is the start time of the lifting motion, and the second vertical broken line is the end time of the lifting motion. As we can see, the force just after start is increasing. This means that the object is pulled in the negative direction of $\mathrm{X}-\mathrm{Z}$ axis. We suppose that a counter-reaction is used for lifting the heavy object. 

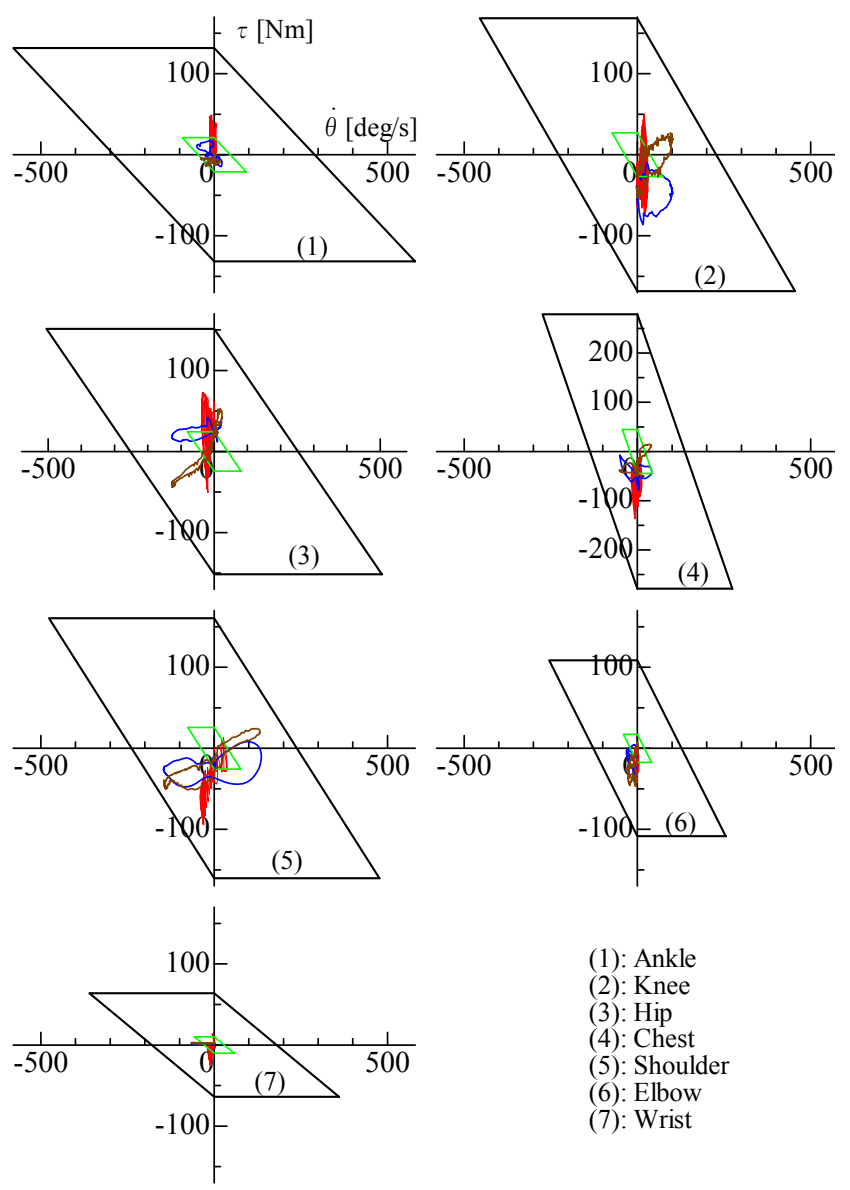

(1): Ankle

(2): Knee

(3): Hip

(4): Chest

(5): Shoulder

(6): Elbow

(7): Wrist

Fig.8. Angular velocity-torque diagram.
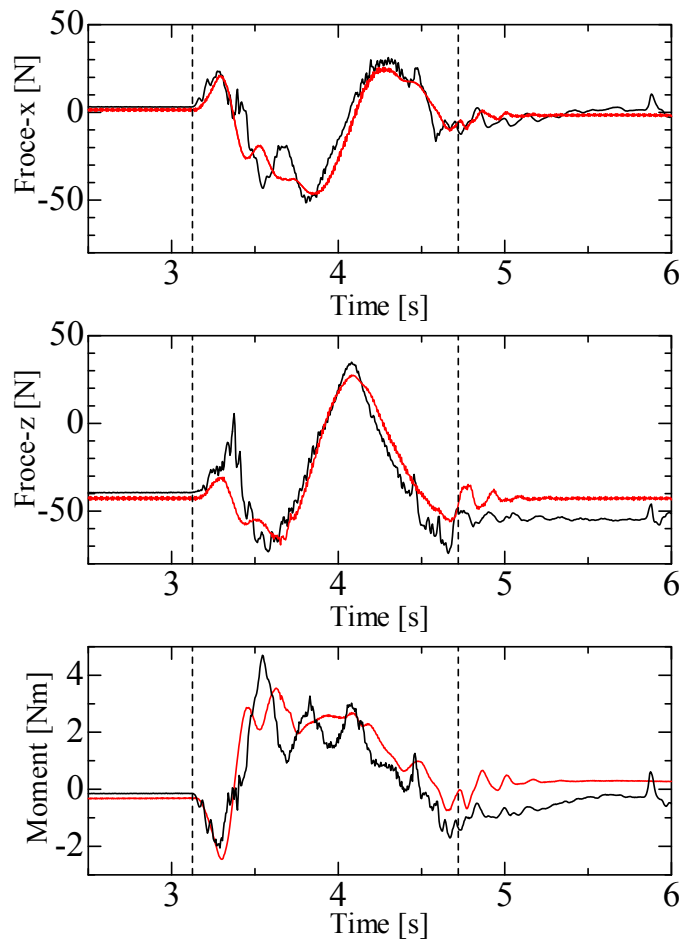

Fig.9. External force and moment at the hand.
After around 3.6s, the force in the z-direction drastically increases. This means the load for the hand is reduced by the acceleration of the object upward. As shown in Fig. 9, both force and moment at the hand are within the allowable load of the force sensor.

The red curve in Fig. 10 shows the change of the ZMP with time. The upper broken line is the position of the toe, the lower broken line is the position of the heel, and the vertical lines are the same as in Fig. 9. Since the ZMP remains within the space delimited by positions of the toe and the heel, then the robot does not tumble. In the figure, ZMP sharply changes at around the end time. We suppose that an impulsive force is applied to the robot when stopping the object. But this oscillation is reduced in a short time by the stabilizer [18] mounted on the robot.

All these results show that our proposed method of lifting heavy objects is effective.

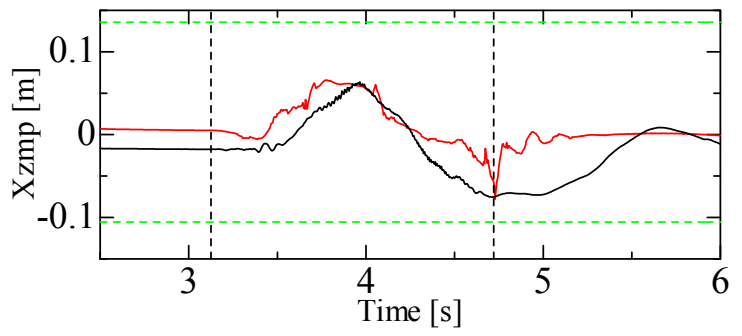

Fig.10. ZMP of the whole system.

Some screenshots of the simulation of lifting the object by the humanoid robot HRP-2 are shown in Fig. 11. From 1) to 2 ) in Fig. 11, the robot tilts backward to accelerate the object upward. When the object gets enough upward speed, the robot slides its own body into under the object. We see that the robot stands up without falling, and changes its posture through a static unstable state and achieves holding the object in a stable way. At the end, the robot stretches its arms to be closer to a singular posture.
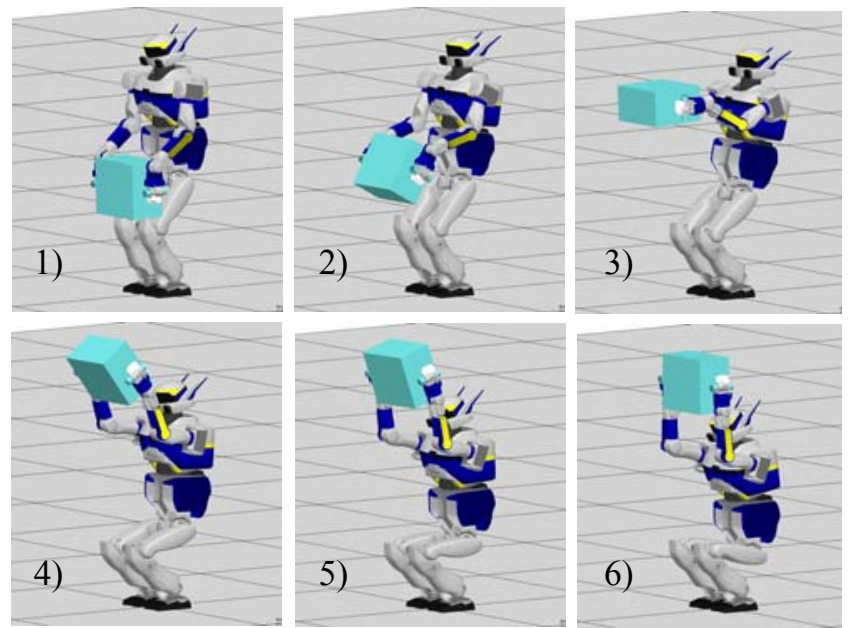

Fig. 11. Lifting motion. 1) initial state, 2) accelerating the object upward, 3) switching motion, 4) sliding into under the object, 5) crouching, 6) final state(sitting). 


\section{Static lifting motion}

For comparison, we simulated the static motion where all joints are controlled to follow the same reference trajectory of the dynamic motion. This motion is 5 times longer than the dynamic motion. In Fig 8, the red curves and the green polygons represent the output of joints and the rated output limitation, respectively. As mentioned in Section III-B, the maximum output limitation is available within only a few seconds $\left(\Delta t_{m o t}=3 \mathrm{~s}\right)$. Since it takes more than $10 \mathrm{~s}$ to finish this static motion, output of joints should be not within the maximum output limitation but within the rated output limitation. But actually output of all joints is saturated as shown in Fig. 8. Hence the static lifting motion cannot be realized in this case.

\section{EXPERIMENT}

We performed experiments of the lifting motion by using the humanoid robot HRP-2 made by Kawada Corp. The number of d.o.f. of HRP-2 is 30 . The height and the weight of the HRP-2 are $\mathrm{h}=1.539 \mathrm{~m}$ and $m_{r o b}=55.3 \mathrm{~kg}$, respectively.

Fig. 12 shows the sequential motion of lifting a heavy object by the HRP-2. Each motion is quite similar to the motion shown in Fig. 11.

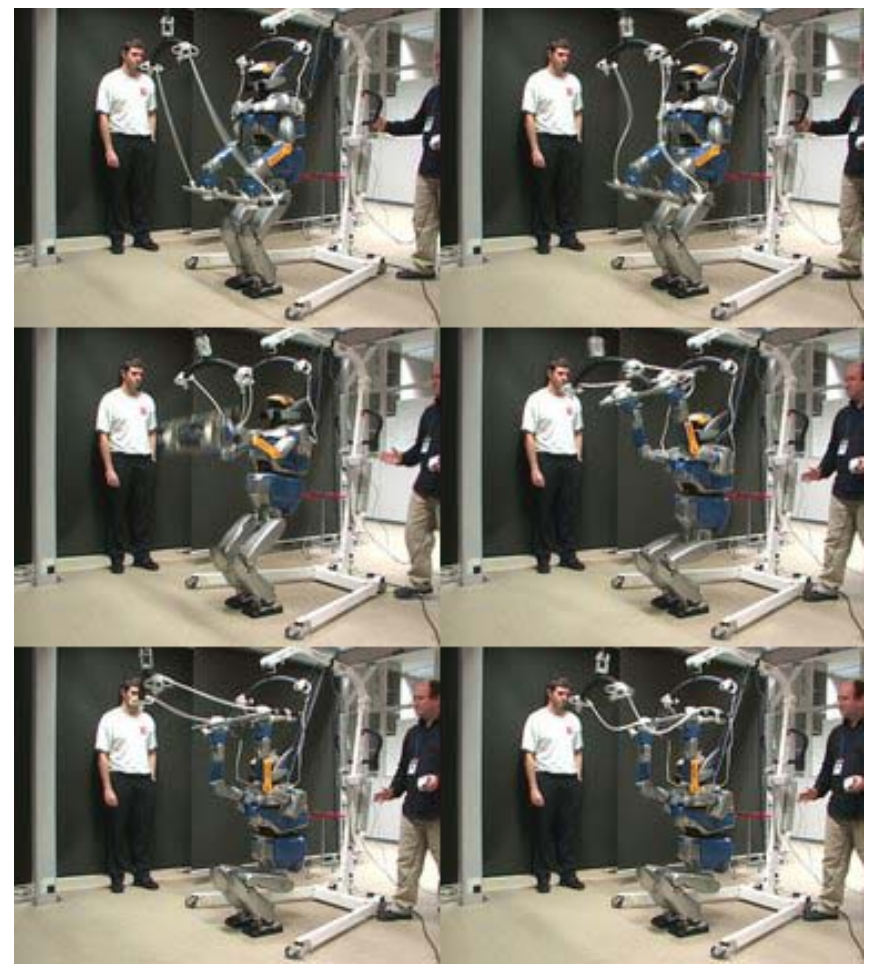

Fig.12. Dynamic lifting by HRP-2.

The brown curves in Fig. 8 show the experimental results of the actuators output. These are not exactly the same as the simulation results shown in Fig. 8, but the shape of the curves is similar to that in the simulation. Besides the output of the low-powered joints, especially the wrist and the elbow, are still controlled to be smaller than that of the other joints.
The black curves in Fig. 9 show the force and the moment that is applied to the hand. Indeed there is noise on the curves in Fig. 9 due to the force sensor, but they are quite similar to the simulation results, the red curves in Fig. 9.

If the motion generated by reducing the allowance of the ZMP is ideal for stability, it should be realized without the stabilizer. To evaluate the proposed motion generator, we do not use the stabilizer in the experiment. As shown in Fig. 13, the ZMP curve changes within the stable area which is delimited by two horizontal broken lines which are the same as Fig. 10. After lifting the object, the ZMP curve slightly oscillates, but it decreases in a short time because of the damping effect of the soles. It shows the practicability of the proposed method from the viewpoint of stability.

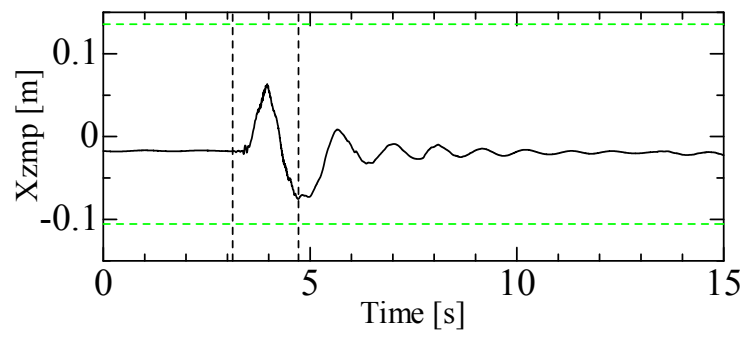

Fig.13. ZMP of the whole system (experimental results).

We challenged a lift of an object of mass $23.4 \mathrm{~kg}$. The motion was obtained by the same way as described in this paper. The successful motion can be seen in the video included in the digital proceedings.

\section{CONCLUSION}

In this paper, we realized the motion of lifting a heavy object dynamically with the humanoid robot.

We firstly examined the static stable reachable area, and show how to select an initial state and a final state of the robot with considering the transition from one singular posture of the arm to another to reduce the load on the arms that have low power outputs. We thus addressed how to move the object from the initial sate to the final one passing through the unstable area in which the robot cannot hold the object statically due to saturation of the actuators. Then, we developed the motion generator based on the optimization method for the dynamic lifting motion. We finally realized the dynamic motion of lifting the heavy object with combining two different kinds of humanoid's motions such as accelerating an object upward and sliding the body into under the object. We verified the effectiveness of the proposed method through simulation and experiments.

\section{ACKNOWLEDGMENT}

This work was supported in part by the Japan Society for the Promotion of Science (JSPS) under Grants-in-Aid for Scientific Research (B). 


\section{REFERENCES}

[1] H. Arai and O. Khatib, "Experiments with Dynamic Skills," Proc. 1994 Japan-USA Symposium on Flexible Automation, pp.81-84, Kobe, Japan, 1994.

[2] K. M. Lynch, N. Shiroma, H. Arai and K. Tanie, "The Roles of Shape and Motion in Dynamic Manipulation: the Butterfly Example," Proc. 1998 IEEE International Conference on Robotics and Automation (ICRA'98), pp.1958-1963, 1998.

[3] K. Yokoyama, H. Handa, T. Isozumi, Y. Fukase, K. Kaneko, F. Kanehiro, Y. Kawai, F. Tomita, and H. Hirukawa, "Cooperative Works by a Human and a Humanoid Robot," Proc. of IEEE International Conference on Robotics and Automation (ICRA'03), pp. 2985-2991, 2003.

[4] T. Takubo, K. Inoue and T. Arai, "Pushing an Object Considering the Hand Reflect Forces by Humanoid Robot in Dynamic Walking," Proc. of IEEE Int. Conf. on Robotics and Automation, pp.1718-1723, 2005

[5] K. Harada, S. Kajita, H. Saito, M. Morisawa, F. Kanehiro, K.Fujiwara, K. Kaneko, and H. Hirukawa, "A Humanoid Robot Carrying a Heavy Object", Proc. of IEEE Int. Conf. on Robotics and Automation, pp. 1724-1729, 2005.

[6] Y. Ohmura, Y. Kuniyoshi, "Humanoid Robot which can Lift a $30 \mathrm{~kg}$ Box by Whole Body Contact and Tactile Feedback," Proc. of the 2007 IEEE/RSJ International Conference on Intelligent Robots and Systems, 2007.

[7] M.Onishi, Z.W.Luo, T.Odashima, S.Hirano, K.Tahara, T.Mukai, "Generation of Human Care Behaviors by Human-Interactive Robot RI-MAN," Proceedings of the 2007 IEEE International Conference on Robotics and Automation, pp.126-127, April 2007.

[8] R. Isaka, R. J. Gregor and S. Kawamura, "Derivation of Reasonable Lifting Movement from Techniques Utilized in Skilled Weightlifter," Transactions of the Japan Society of Mechanical Engineers, vol.64, no.623, pp.119-126, 1998.

[9] N. Matsunaga and S. Kawaji, "Motion Analysis of Human Lifting Works with Heavy Objects," Journal of Robotics and Mechatronics, vol.17, no.6, pp.628-635, 2005.

[10] C. Wang, W. Timoszyk and J. Bobrow, "Payload Maximization for Open Chained Manipulators: Finding Weightlifting Motions for A Puma 762 Robot" IEEE Trans. on Robotics and Automation, vol. 17, no. 2, pp.218-224, 2001.

[11] Arisumi, H., Chardonnet, J-R., Kheddar, A., and Yokoi, K., "Dynamic Lifting Motion of Humanoid Robots," Proc. of the IEEE International Conference on Robotics and Automation (ICRA'07), pp. 2661- 2667, 2007.

[12] K. Akachi, T. Isozumi, M. Hirata, S. Ohta and M. Ishizaka, "Development of the Humanoid Robot, HRP-2," KAWADA Technical Report, vol.23, pp. 20-25, 2004.

[13] Arisumi, H., Yokoi, K., Komoriya, K., "Dynamic Performance Characterization of Manipulators under Actuator Constraints," Proc. of the International Conference on Recent Advances in Mechatronics (ICRAM'95), pp.1042-1047, 1995

[14] A. Wachter, L.T. Biegler, "On the Implementation of a Primal-Dual Interior Point Filter Line Search Algorithm for Large-Scale Nonlinear Programming”, Mathematical Programming, vol. 106, pp. 25-57, 2006.

[15] S. Miossec, K. Yokoi, A. Kheddar, "Development of a software for motion optimization of robots - Application to the kick motion of the HRP-2 robot", Proceedings of the 2006 IEEE International Conference on Robotics and Biomimetics, 2006.

[16] http://www.maxonmotor.com/de_motor.asp

[17] J-R. Chardonnet, S. Miossec, A. Kheddar, H. Arisumi, H. Hirukawa, F. Pierrot, K. Yokoi, "Dynamic Simulator for Humanoids Using Constraint-Based Method with Static Friction," Int. Conf. on Robotics and Biomimetics, pp. 1366-1371, 2006.

[18] K. Kaneko, et al., "Stabilizer for biped walking," Proc. of the $21^{\text {st }}$ Annual Conference of the Robotics Society of Japan, 3A34, 2003. (in Japanese) 